\title{
Primary Central Nervous System Lymphoma with Exclusive Leptomeningeal Involvement: The Diagnostic Challenge of a Rare Presentation of an Uncommon Disease
}

Miguel Santos, Tatiana Pires, Ana Rodrigues, Adriana Bandeira, Ana Albuquerque, Flávia Santos, Miriam Magalhães, Maria Jesus Banza

Department of Internal Medicine, Centro Hospitalar de Leiria, Leiria, Portugal

\section{Doi: 10.12890/2022_003107 - European Journal of Case Reports in Internal Medicine - @ EFIM 2022}

Received: 06/12/2021

Accepted: $12 / 01 / 2022$

Published: $27 / 01 / 2022$

How to cite this article: Santos M, Pires T, Rodrigues A, Bandeira A, Albuquerque A, Santos F, Magalhães M, Banza MJ. Primary central nervous system lymphoma with exclusive leptomeningeal involvement: the diagnostic challenge of a rare presentation of an uncommon disease. EJCRIM 2022;9:

doi:10.12890/2022_003107.

Conflicts of Interests: The authors declare there are no competing interests.

This article is licensed under a Commons Attribution Non-Commercial 4.0 License

\section{ABSTRACT}

Primary central nervous system lymphoma (PCNSL) is a rare and aggressive extra-nodal non-Hodgkin lymphoma (NHL). It must be confined to the brain, eyes, spinal cord or leptomeninges without systemic involvement at the time of diagnosis. Disease confined to the cerebrospinal fluid (CSF) is a rare form of presentation and poses a particular diagnostic challenge.

We present the case of an 82-year-old man admitted to hospital because of an acute confusional state, later revealed to be due to PCNSL with exclusive leptomeningeal involvement. The diagnostic process was further impaired (or, perhaps, aided?) by the onset of a COVID-19 outbreak on the ward.

\section{LEARNING POINTS}

- Primary central nervous system lymphoma is a rare and aggressive form of non-Hodgkin lymphoma.

- Exclusive involvement of the cerebrospinal fluid (CSF) is a rare form of presentation of PCNSL.

- The diagnosis is usually histopathological but, in the absence of a solid lesion that can be biopsied, CSF cytology and flow cytometry may be sufficient for a definitive diagnosis.

\section{KEYWORDS}

Primary central nervous system lymphoma, leptomeningeal lymphoma

\section{CASE DESCRIPTION}

An 82-year-old man presented to the Emergency Department with acute onset confusion starting the previous day. According to a family member, the patient had been complaining of sudden loss of hearing and decreased visual acuity, both lasting a few days. He had also referred pain in his lower back, and decreased strength in his right lower limb with a few weeks' duration, but thought nothing of it as he thought it was probably sciatica, which he had had before. There was no history of fever.

Before this episode, he was a fully autonomous person, still working part-time as a businessman. He had a previous medical history of hypertension, hypercholesterolemia and benign prostatic hyperplasia and took regular medication for these conditions, including an angiotensin receptor blocker/hydrochlorothiazide combination, tamsulosin and a statin.

At hospital admission, the patient was disoriented but able to obey simple commands, although communication was difficult due to marked hypoacusia. There appeared to be decreased visual acuity in the left eye and muscular strength in the right lower limb was diminished. 
He was unable to walk at this point. There were no other remarkable changes on physical or neurological examination.

Blood work was unremarkable except for moderate hyponatremia (sodium of $126 \mathrm{mmol} / \mathrm{l}$ ). He underwent head CT, which was also unremarkable, and lumbar spine CT which revealed several degenerative changes with slight compression of the nerve roots at L4-L5 and L5-S1 levels. This could explain the sciatica and was a possible cause of the loss of strength in the right lower limb, but the confusion remained unexplained. It was assumed at this stage it could be due to the hyponatremia which could, in turn, be secondary to the hydrochlorothiazide that the patient took for hypertension. He stayed in the emergency department under observation and for correction of hyponatremia.

After the hyponatremia was corrected, there was no improvement in the patient's mental status. A lumbar puncture was then performed. The lumbar puncture was traumatic. However, even when this was taken into consideration, the CSF presented with 960 white blood cells, with $83 \%$ polymorphonuclear cells, proteins $1341.3 \mathrm{mg} / \mathrm{l}$ and hypoglycorrhachia.

The diagnosis of bacterial meningitis was assumed at this point. The CSF was negative for a panel of common bacterial meningitis antigens (including Haemophilus influenzae, Neisseria meningitidis, Streptococcus pneumonia and Streptococcus agalactiae). Further samples of CSF were taken for bacteriological examination and culture and the patient was started on antibiotics (ceftriaxone, ampicillin and vancomycin) and admitted to an Internal Medicine ward.

After admission he was seen by Ophthalmology and an ENT specialist. Both examinations were unremarkable.

After a few days of antibiotic therapy, there was no clinical improvement. The CSF cultures came back negative, which prompted a review of the established diagnosis.

A brain MRI and EEG were both unremarkable and the patient was tested for HIV, which was negative. A repeat lumbar puncture was performed. This time, the lumbar puncture was not traumatic and clear CSF was drawn. The hyperproteinorrhachia and hypoglycorrhachia were still present, but the cells observed indicated a different diagnosis: there were 2560 white blood cells, of which $11 \%$ were monocytes, there were no polymorphonuclear cells and the remaining cells were large cells with basophilic irregular cytoplasm and an elevated nuclei to cytoplasm ratio. These cells were suspected to be immature or clonal lymphocytes and the diagnostic hypothesis of central nervous system lymphoma was first discussed at this point. CSF samples were sent for cytology and flow cytometry and the patient underwent CT of the chest, abdomen and pelvis, looking for other areas suspicious for involvement by lymphoma. There were no remarkable findings on this CT. At this time, there was a COVID-19 outbreak on the ward. The patient also tested positive and was transferred to a COVID-19 ward. As he developed symptoms of COVID-19, he was started on dexamethasone. Curiously, his confusion finally resolved after starting corticotherapy. The CSF cytometry came back with 95\% B-lymphocytes expressing CD19, CD38, CD200, CD79b, CD43, CD10, CD11c and CD20 and negative for CD5, CD23, CD25, CD305 and CD103 surface antigens. These findings are compatible with a diffuse large B-cell lymphoma and the final diagnosis of PCNSL was made.

After recovering from COVID-19, the patient was discharged from hospital and referred to Haematology for chemotherapy. He was about to be started on a methotrexate-based chemotherapy regimen when he was re-admitted to hospital with fever and dyspnoea. He died a few days later of hospital-acquired pneumonia.

\section{DISCUSSION}

This case showcases the diagnostic challenge posed by a rare illness such as PCNSL. Indeed, with an incidence of 0.43 cases per 100,000 people ${ }^{[1]}$, PCNSL is not usually among the first diagnostic hypotheses considered in a case of acute confusional state, particularly in an HIVnegative patient. The incidence of PCNSL is 1000 times higher in HIV patients than in the general population ${ }^{[2]}$.

In this instance, the final diagnosis was particularly challenging as there were no changes on brain imaging (CT and MRI). PCNSL usually presents as a solid brain lesion with characteristic features on MRI, namely the presence of a solitary brain lesion with homogeneous contrast enhancement and well-defined borders, a low signal on T2-weighted MRI and restricted diffusion on diffusion-weighted MRI ${ }^{[3]}$. Isolated CSF involvement, as in this case, is a rare form of presentation of this rare disease ${ }^{[4]}$.

This patient was simultaneously very unfortunate and very lucky, as he was caught up in a COVID-19 outbreak in the hospital. On one hand, this event further delayed the final diagnosis and treatment. However, it was after contracting COVID-19 that the patient finally improved regarding his mental status as he was given corticosteroids as treatment for COVID-19.

Indeed, PCNSL are typically steroid responsive, although nowadays this is no longer a mainstay of treatment ${ }^{[4]}$. PCNSL was suspected (but not confirmed) at the time the patient contracted COVID-19. The fact that his neurological state improved under steroid therapy was another positive clue in favour of this diagnosis; however, steroid therapy should not be started routinely in similar cases, especially before a definitive histopathological diagnosis is achieved, as these results may be inconclusive after steroid therapy ${ }^{[4]}$. In most cases of PCNSL, the final diagnosis will be achieved by stereotactic brain biopsy. However, in cases such as the one presented, in the absence of a solid lesion that can be biopsied, CSF cytology and flow cytometry may be sufficient for a definitive diagnosis ${ }^{[5]}$. 


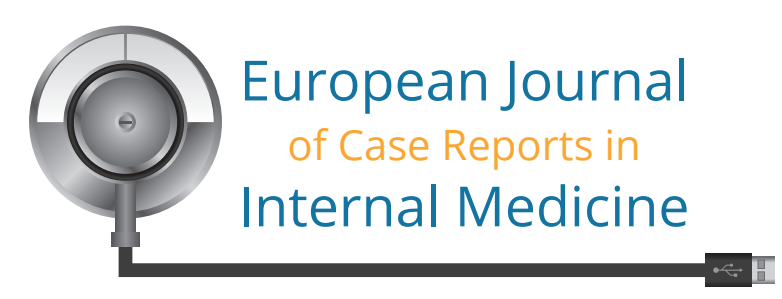

This patient was referred to Haematology for chemotherapy. The standard of care for elderly patients with PCNSL is not well established. However, it is recognized that these patients have a higher risk of neurotoxicity and whole brain radiotherapy should be avoided for this reason. Methotrexate-based chemotherapy regimens appear to lead to better outcomes ${ }^{[4]}$.

Even under adequate treatment, the prognosis for PCNSL is poor in elderly patients, with a median overall survival time of 1.1-3.2 years ${ }^{[6]}$. As interesting as this case is in itself, the main lesson derives from its complexity and the importance of always reviewing the established diagnosis, especially when there are symptoms that remain unexplained and/or the patient does not respond as expected to treatment.

\section{REFERENCES}

1. Ostrom QT, Gittleman H, de Blank PM, Finlay JL,Gurney JG,McKean-Cowdin R,et al. American Brain Tumor Association adolescent and young adult primary brain and central nervous system tumors diagnosed in the United States in 2008-2012. Neuro Oncol 2016;18(Suppl 1):i1-i50.

2. Flinn IW, Ambinder RF. AIDS primary central nervous system lymphoma. Curr Opin Oncol 1996;8:373.

3. Nabavizadeh SA, Vossough A, Hajmomenian M, Assadsangabi R, Mohan S. Neuroimaging in central nervous system lymphoma. Hematol Oncol Clin North Am 2016;30:799-821. $\mathrm{Han}$ CH, Batchelor TT. Diagnosis and management of primary central nervous system lymphoma. Cancer 2017;15:4314-4324.

Fischer L, Martus P, Weller M,KlasenHA,RohdenB,RöthA, et al. Meningeal dissemination in primary CNS lymphoma: prospective evaluation of 282 patients. Neurology 2008;71:1102-1108.

6. Abrey LE, Ben-Porat L, Panageas KS,YahalomJ,BerkeyB,CurranW, et al. Primary central nervous system lymphoma: the Memorial Sloan-Kettering Cancer Center prognostic model. J Clin Oncol 2006;24:5711-5715. 